Revue

de Sémantique

et Pragmatique

\section{Revue de Sémantique et Pragmatique}

41-42 | 2017

Cadrage sur la variation, le changement lexical et le changement grammatical en français actuel

Les constructions comme variables pour la variation syntaxique et le rôle des traditions discursives dans la production des variantes

Henri-José Deulofeu

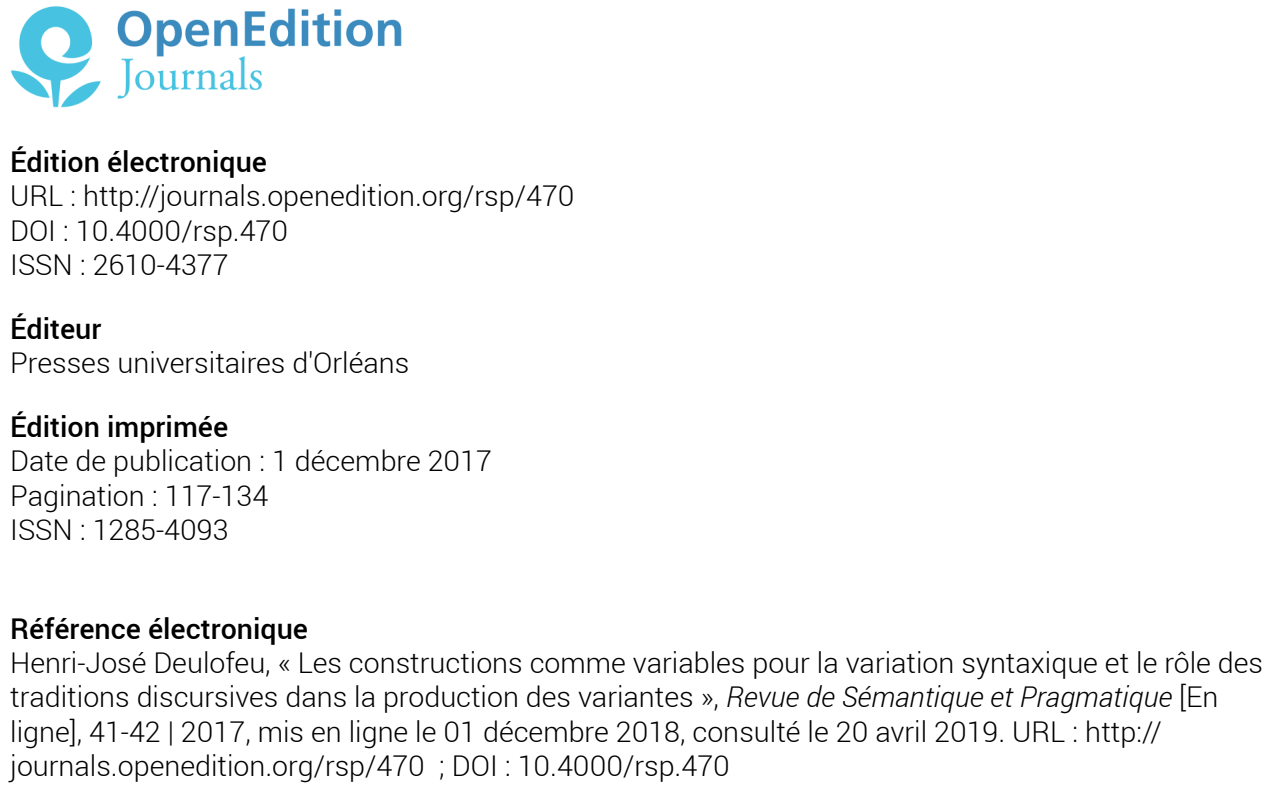

Revue de Sémantique et Pragmatique 


\title{
LES CONSTRUCTIONS COMME VARIABLES POUR LA VARIATION SYNTAXIQUE ET LE RÔLE DES TRADITIONS DISCURSIVES DANS LA PRODUCTION DES VARIANTES
}

\author{
Henri-José Deulofeu \\ Laboratoire d'informatique fondamentale \\ Université d'Aix-Marseille
}

L'objectif de cet article est de réexaminer la notion d'invariant sémantique

on syntaxique du français « tout court », toutes variétés confondues, il était

L'objectif 
s aux référents et donc,

par définition, ne « disent pas la même chose ». Le sens n'est pas préexistant au

s, ce qui rend plus complexe encore

invariante définie linguistiquement. L'invariant peut être établi

es

sions sur le «sa 
le avec

cture sémantique

ssé composé. La variable est définie comme la notion de «passé accompli ». On est donc pour cette perspective dans un e variantes libres : « For certain identifiable 
endre compte des faits d'alternance sur une base différente : «Conformément

de Vaugelas : «

ment synchro

orpus disponibles

à cette époque rend sans doute difficile de trancher empiriquement entre les deux hypothèses, mais la discussion souligne le danger de définir les variables de la variation syntaxique dans les termes d'une analyse sémantique superfi ue montre que l'apparente variation

fisamment nombreuses permettaient d'affirmer que

ignificative entre deux auxiliaires et l'autre ayant perdu

ut degré « absolu ». Pour le haut degré relatif, deux adverbes ( 
a tendance à l'organisation par terme couvrant se manifeste $f:$ dans (3)

« même sens » révèle en fait un répertoire différent de 
ée ». Selon ce principe, il

tion d'un clitique objet direct - animé sur le modèle du substrat franco-provençal (7), sont ignorées au profit ais. Au lieu de reconnaitre une op

riation syntaxique », là où il faudrait reconnaître la présence de distinctes comportant une association spé 
ajoutées. Deulofeu (2001) présente

aine français, outre les travaux du Gars sur la « grammaire seconde », par

os églises détruites » [...] plutôt que « Nous n'oublierons jamais la violation de la Belgique, l'invasion de notre sol, la destruction de nos églises » [...] ou que « Nous n'oublie

é, celui de la forme des «propositions» subordonnées. Pour l'expression d'un contenu qui est posé comme signifiant une même «situation », l'auteur affiche une préférence pour une tradition que

rappelant la doxa 
e à pétrole est un ustensile d'éclairage. 2. Elle se compose

$\mathrm{n}$ - donc apparemment vous l'utilisez comme un modificateur de per sations que vous avez habi

cente à ces effets de variation. 
$\mathrm{t}$ les rap

ues.

3.2.1 Relation d'héritage entre constructions : Michaelis \& Lambrecht (1996)

utre hériter des propriétés de plusieurs constructions moins spécifiées.

ais

en termes

ecteurs sous-spécifiés 
ous-spécifiées et que, d'autre part, des locuteurs peu scolarisés peuvent employer des formes sur-spécifiées quand ils

, le système de la langue française autorise aussi bien les prépositions sous-spécifiées que les prépositions plus spécifiées séman

rtefact français standard écrit, a éliminé les formes sous-spécifiées en les considérant comme de simple signifiants fautifs. Dans l'expression spon

s préjudice du verglas

tion par certains 
st en fait « «favorable » à tous les styles, dès lors que le souci de précision n'est pas un enjeu de communication. La construction à préposition sous-spé fiée et les constructions plus spécifiées n'expriment évidemment pas la même

erbe », en y ajoutant les traits sémantiques de la préposition. Ce modèle d'utilisation res

ans les rela

on « rela

milieu professionnel dans lequel j'évolue, le secteur dans lequel je tra

réellement dans la langue parlée. » 
possibilités offertes par le système en fonction des objectifs de communication : être précis ou rester dans l'indétermination sémantique, que ce soit à des fins de simplicité ou à des fins esthétiques. Et des traditions discursives où l'on s'efforce d'être toujours le plus précis possible, dans la ligne des recommanda

is, à l'instar de leurs

sécutive » est un modifieur de $\mathrm{N}$ commandée par la présence d'un trait [+ qualité] dans 
ais est porté par l'article indéfini. La

r la base d'une même signification référentielle,

iversel

u comme une simplification « moderne » du système des conjonctions, est en fait une variante possible de subordination que l'on retrouve dans l'ancienne langue : « [En ancien français] les articulations sus

n de subordination, c'est-à-dire de modification d'une tête ver tape ultime de la sous-spécification étant celle où le morphème est un 
$\mathrm{r}$ les analyses de

jeure entre les deux usages apparaît dans la

eudo clivées d « transition» 
'est la responsable qui qui

ifficiles

comme je veux les autres je m'en fiche

eux

seiller en « addition de considéra

teur de discours à lui tout seul. On peut ajouter que dans la variété méridionale, si

plus haut, 
par les locuteurs

ropre au fran

on de variable syntaxique

me, par exemple l'emploi sous-specifié 
entrée sur

e l'autre, le domaine des auxiliaires »,

erspective linguistique et sociolinguistique dans l'étude des rela

$s$ linguistiques en

rammaticale : le

cas des prépositions ; hypothèses linguistiques et sociolinguistiques », 
ites avec

lo Andrée, Vuillaume

tional self : theorizing dia

as a symptom

rechet, K. (1996), « Toward a Construction-Based Model of Language Function: The Case of Nominal Extraposition »

syntactic variation », in Newmeyer F.J. 